\title{
3 Research Square

\section{Are preoperative high soluble programmed death ligand 1 levels responsible for patients' poor prognoses in hepato-biliary-pancreatic cancer?}

Rei Okada

Toho Daigaku https://orcid.org/0000-0003-2369-4642

Yuichiro Otsuka

Toho Daigaku

Masaru Tsuchiya

Toho Daigaku

Tetsuya Maeda

Toho Daigaku

Jun Ishii

Toho Daigaku

Yoshihisa Kubota

Toho Daigaku

Kimihiko Funahashi

Toho Daigaku

Hironori Kaneko

Toho Daigaku

Hideaki Shimada ( $\square$ hideaki.shimada@med.toho-u.ac.jp )

Toho Daigaku https://orcid.org/0000-0002-1990-8217

\section{Research}

Keywords: programmed death-ligand 1, hepato-biliary-pancreatic cancer, prognosis

Posted Date: August 5th, 2020

DOI: https://doi.org/10.21203/rs.3.rs-52812/v1

License: (c) (i) This work is licensed under a Creative Commons Attribution 4.0 International License.

Read Full License 


\section{Abstract \\ Background}

Several reports showed that high soluble programmed death-ligand 1(SPD-L1) level was a risk factor for poor prognosis in various tumors. To date, the clinicopathologic and prognostic impact of SPD-L1 level in patients with hepato-biliary-pancreatic cancer have not been determined.

\section{Methods}

A total of 119 patients (66 patients with hepatocellular carcinoma, 23 patients with cholangiocarcinoma, 30 patients with pancreatic cancer) who were treated at the Toho University Omori Hospital (Tokyo, Japan) from 2008 to 2016 were retrospectively analyzed. SPD-L1 levels were measured using an enzymelinked immunosorbent assay for PD-L1 to evaluate clinicopathologic and prognostic impact.

\section{Results}

SPD-L1 levels were significantly higher in low-albumin group than normal albumin group. According to stages in hepatocellular carcinoma and cholangiocarcinoma, there were no significant differences in SPD-L1 levels, which gradually increased according to stage in pancreatic cancer. Using a cut-off value of $81.6 \mathrm{pg} / \mathrm{ml}$ for SPD-L1 level, the high SPD-L1 group showed significantly worse prognosis than the low SPD-L1 group in patients with pancreatic cancer. Multivariate analysis identified SPD-L1 level $\geq$ $81.6 \mathrm{mg} / \mathrm{dl}(p=0.047)$ as an independent predictor of poor overall survival in patients with pancreatic cancer.

\section{Conclusion}

High SPD-L1 levels were independently associated with poor prognosis. However, this association in hepatocellular carcinoma or cholangiocarcinoma was not clear.

\section{Background}

Programmed death-ligand 1 (PD-L1) is an immune checkpoint protein within the cancer-immunity cycle that is expressed on the surface of tumor cells and tumor infiltrating immune cells to downregulate T-cell function [1]. Recently, soluble PD-L1 (sPD-L1) has been detected in the blood of cancer patients [2]. Several clinical studies have evaluated prognostic values of SPD-L1 in patients with various cancers and explored the associations of SPD-L1 levels with clinicopathologic factors [3-5]. As a result, some reports showed that high SPD-L1 level was a risk factor for poor prognosis in various tumors including non-small cell lung cancer, gastric cancer, hepatocellular carcinoma (HCC), and renal cell cancer $[3,5-10]$. 
In patients with HCC, high SPD-L1 level and high PD-L1 expression in tumors has been identified as a poor prognostic factor $[5,11]$. In patients with cholangiocarcinoma (CCA), high PD-L1 expression in tumors has been identified as a poor prognostic factor [12]. On the other hand, the clinicopathologic significance of high SPD-L1 level has not been reported. In patients with pancreatic cancer (PC), high SPDL1 level was not significantly associated with overall survival [13]. To date, clinicopathological and prognostic impact of SPD-L1 levels in patients with hepato-biliary-pancreatic cancer have not been determined $[4,5,14]$.

Therefore, we analyzed cross-sectional SPD-L1 levels of patients with hepato-biliary-pancreatic (HBP) cancer and assessed the relationship with clinicopathologic factors and prognosis.

\section{Methods}

\section{Patients}

A total of 119 patients ( 66 patients with HCC, 23 patients with CCA, 30 patients with PC) who were treated at the Toho University Omori Hospital (Tokyo, Japan) from 2008 to 2016 were retrospectively analyzed. Among the 66 patients with HCC, 19 patients had stage I, 14 had stage II, 28 had stage III, and 5 had stage IV. Among 23 patients with CCA, 9 patients had stage I, 10 had stage II, 1 had stage III, and 3 had stage IV. Among 30 patients with PC, 4 patients had stage I, 15 had stage II, 4 had stage III, and 7 had stage IV. All patients were treated with liver resection, pancreaticoduodenectomy, bile duct resection, distal pancreatectomy, or examination laparotomy. The 15 patients with stage IV disease included distant lymph node metastasis $(n=2)$, organ metastasis $(n=4)$, and peritoneal metastasis or cancer cells on peritoneal cytology $(n=9)$. Using the tumor-node-metastasis (TNM) classification system of malignant tumors of the International Union for Cancer Control (IUCC), 8th edition [15], the final stage of HBP cancer was assessed pathologically.

This retrospective study was approved by the Institutional Ethics Committee of the Toho University (IRB no. A18103). All patients were followed-up until the end of December 2019 or death.

\section{Sample collection and enzyme-linked immunosorbent assay}

All serum samples were collected before surgery, and SPD-L1 levels were measured using an enzymelinked immunosorbent assay (ELISA) for PD-L1 (R\&D Systems, Inc., Minneapolis, MN, USA) as previously described [3].

\section{Study design and serum biomarker analysis}

In order to evaluate the relationship with SPD-L1 level, C-reactive protein (CRP), albumin, and plasma fibrinogen were analyzed before surgery. White blood cell, neutrophil, and lymphocyte counts were also analyzed. Cut-off values were determined in accordance with the institutional standards for white blood 
cell count $\left(7000\right.$ cells $\left./ \mathrm{mm}^{3}\right)$, CRP levels $(0.3 \mathrm{mg} / \mathrm{mL})$, and albumin $(3.5 \mathrm{mg} / \mathrm{dl})$. Then, we analyzed the association between SPD-L1 level and clinicopathologic factors with HBP cancer.

\section{Statistical analyses}

Statistical analysis was performed using JMP statistical software (version 12; SAS Institute, Cary, NC, USA). Serum biomarker levels were expressed as the mean \pm standard deviation. Comparisons between unpaired groups for these variables were conducted with the Mann-Whitney Utest. In addition, the survival rate was calculated by the Kaplan-Meier product limit estimate. Differences between groups regarding survival were analyzed by the log-rank test. Multivariate analysis using the Cox proportional hazards model was used to assess significant predictors identified by univariate analysis. We considered $p<0.05$ as statistically significant.

\section{Results}

\section{Correlation and association of serum programmed death ligand 1 level with serum biomarkers}

The associations of SPD-L1 levels with several blood tests were evaluated (Table 1). SPD-L1 level was significantly higher in the low-albumin group than in the abnormal albumin group ( $p=0.035$, Table 1$)$. There were no significant differences between SPD-L1 levels and serum biomarkers in the other parameters: white blood cell, neutrophils, lymphocytes, C-reactive protein, total bilirubin, and amylase.

\section{Comparison of soluble programmed death ligand 1 levels of hepato-biliary-pancreatic cancer and each TNM stage}

The median SPD-L1 level for 66 patients with HCC, 23 patients with CCA, and 30 patients with PC was 66.9 (range 15.8-188.1) pg/ml, 64.5 (range 26.4-138.5) pg/ml and 62.6 (range 36.7-147.6) pg/mL (Fig. 1a). There were no significant differences in SPD-L1 levels among HBP cancer (HCC vs CCA $p=$ 0.895 , HCC vs PC $p=0.818$, CCA vs PC $p=0.686$ ). There were no significant differences in SPD-L1 levels according to UICC stages in HCC and CCA (Fig. 1b, 1c). On the other hand, SPD-L1 levels gradually increased according to UICC stage in PC (Fig. 1d). SPD-L1 levels of stage IV PC was significantly higher than that of stage $\mathrm{I} / \mathrm{II} / \mathrm{III} \mathrm{PC}$ (Fig. 1c, $\mathrm{p}=0.039)$.

Comparisons of soluble programmed death ligand 1 levels according to clinicopathologic factors and various biomarkers with HBP cancer

SPD-L1 levels were not associated with any of the clinicopathologic factors, such as gender, age, stage, tumor size, and conventional tumor markers, in patients with HCC and CCA (Table 2a, 2b, 2c). Except, SPD-L1 levels were significantly higher in PC patients with high CA19-9 levels ( $p=0.028$, Table 2c). 
Overall survival curves according to soluble programmed death ligand 1 levels in the patients with HBP cancer

All 119 cases were divided by quartiles according to SPD-L1 levels as follows: the range of SPD-L1 levels with Q1 was $15.8 \mathrm{pg} / \mathrm{ml}$ to $49.3 \mathrm{pg} / \mathrm{ml}$, Q2 was $49.8 \mathrm{pg} / \mathrm{ml}$ to $64.3 \mathrm{pg} / \mathrm{ml}$, Q3 was $64.5 \mathrm{pg} / \mathrm{ml}$ to $81.5 \mathrm{pg} / \mathrm{ml}$, and Q4 was $81.7 \mathrm{pg} / \mathrm{ml}$ to $188.0 \mathrm{pg} / \mathrm{ml}$. Although there were no significant differences between each group ( $p=0.878)$, the Q4 group showed the worst survival rate (Fig. $2 \mathrm{a})$. Therefore, we decided the cut-off value of $81.6 \mathrm{pg} / \mathrm{ml}$ SPD-L1 level to evaluate prognostic significance of SPD-L1 in further analyses. Although the Q4 group showed worse prognosis than the other groups (Q1Q2Q3), the difference was not statistically significant ( $p=0.333$, Fig. $2 b$ ).

In order to evaluate the prognostic significance of SPD-L1 in each cancer type, overall survival (OS) was compared between Q1Q2Q3 group vs Q4 group in each cancer types (Fig. 3). There was no prognostic significance in $\operatorname{HCC}(p=0.977$, hazard ratio $=1.017$, Fig. $3 a)$ and CCA $(p=0.665$, hazard ratio $=0.717$, Fig. 3b). On the other hand, Q4 group showed significantly worse prognosis than Q1Q2Q3 group in patients with $\mathrm{PC}(p=0.005$, hazard ratio $=5.059$, Fig. $3 c)$.

\section{Univariate and multivariate analysis of risk factors for overall survival with pancreatic cancer}

Several prognostic factors, including SPD-L1 levels, were evaluated in patients with PC (Table 3). The univariate analysis identified the UICC M1, and SPD-L1 level $\geq 81.6 \mathrm{mg} / \mathrm{dl}$ as significant predictors of poor OS. Moreover, multivariate analysis conducted using Cox proportional hazard regression model identified the UICC M1 ( $p=0.017$; HR, 3.997; 95\% Cl: 1.779-12.03), and SPD-L1 level $\geq 81.6 \mathrm{mg} / \mathrm{dl}$ ( $p=$ 0.047 ; HR, 3.588; $95 \% \mathrm{Cl}: 1.419-11.19)$ as independent predictors of OS.

\section{Discussion}

In the present study, preoperative SPD-L1 levels were analyzed in a total of 119 patients with HCC, CCA, or PC. In all 3 cancer types, high SPD-L1 levels were significantly associated with low-albumin levels. In patients with PC, high SPD-L1 levels were associated with high CA19-9 levels but not with tumor stage. Using a cut-off value of $81.6 \mathrm{pg} / \mathrm{ml}$, high SPD-L1 level was an independent risk factor for poor OS of patients with PC.

SPD-L1 levels have been reported to increase in patients with cancer and/or systemic inflammation in HCC, gastric cancer and PC [3-5]. Although previous studies showed the significant association between SPD-L1 and CRP, there was no significant relationship between SPD-L1 level and CRP in our current study. Such discrepancy could be partly explained by the fact that there was big difference between the ratio of stage IV in previous studies and the ratio in our present study: stage IV cases accounted for $14 \%$ $85 \%$ in previous studies and $12 \%$ in our study. In stage IV disease, the tumor microenvironment contains many cells producing inflammatory cytokines and promotion of metastatic disease. 
Although previous reports did not show that high SPD-L1 levels had significant negative effect on OS in PC, our present data showed that high SPD-L1 level was a significant risk factor reducing patients' survival. A discrepancy such as this could be explained by the aforementioned ratio of stage IV disease in previous reports being higher than in our present study. Too much stage IV disease might mask prognostic impact of PD-L1 expression and the effects of checkpoint blockade in PC $[16,17]$. In other words, PD-L1 blockade may effectively improve the prognosis of resectable PC with high PD-L1 levels. Given the small number of patients included in our study, further studies are necessary to confirm this observation.

Although SPD-L1 level was significantly associated with poor prognosis $[5,11]$ in previous reports in HCC, our present series did not show such association. Two previous reports used different ELISA kits than the one used in our present analysis. Moreover, the detection limits and the cut-off values were completely different from those in our series. Such differences might partly explain these discrepancies. Regarding the correlation between high SPD-L1 level and high CRP, 2 previous reports showed positive correlations, in HCC [5] and in PC [4]. They suspected that an increased activation of innate immunity is part of the immunosuppressive environment which hampers activity of the adaptive anti-tumor response. We could not confirm such correlations in our present study. The impact of SPD-L1 levels might be independent to the activity of inflammatory cytokines in HCC.

One of the limitations of the present study was the lack of healthy controls to compare sPD-L1 levels with those of patients with HBP cancer. Using the same ELISA kit that was used in the present study, Chen et al reported that the median serum PD-L1 level in healthy controls was $48.15 \mathrm{pg} / \mathrm{ml}$ [18]. The median serum PD-L1 level reported in our present study was slightly higher than this value. Secondly, no immunohistochemical analysis was performed to evaluate the impact of tissue PD-L1 expression on serum PD-L1 levels. In cancer, the relationship between SPD-L1 and PD-L1 expression in tissues remains unclear. SPD-L1 may be produced by multiple sources via distinct mechanisms from both tumor and immune cells $[19,20]$.

\section{Conclusion}

High SPD-L1 levels might be associated with tumor progression in PC. Moreover, high SPD-L1 levels were independently associated with poor patient survival in the PC group. However, these associations in HCC and CCA were limited.

\section{Abbreviations}

PD-L1: Programmed death-ligand 1; HCC: Hepatocellular carcinoma; CCA: Cholangiocarcinoma; PC:

Pancreatic cancer; AFP: a-fetprotein; PIVKA-II: Protein Induced by Vitamin K Absence-II; CEA:

Carcinoembryonic antigen; CA19-9: Carbohydrate antigen 19-9; CRP: C-reactive protein

\section{Declarations}




\section{Availability of data and materials}

All date used and analyzed during this study are available from the corresponding author on reasonable request.

\section{Ethics approval and consent to participate}

This study was approved by the Institutional Ethical Committee of the Omori Medical Center, Toho University School of Medicine (Tokyo, Japan). All patients provided written informed consent for the sampling, analyses, and publications.

\section{Consent for publication}

Written informed consent was obtained from the patient and legal guardian for publication of these reports.

\section{Competing interests}

The authors declare that they have no competing interests.

\section{Funding}

This research was supported by the Project for Cancer Research and Therapeutic Evolution (P-CREATE) from the Japan Agency for Medical Research and Development, AMED.

\section{Authors' contributions}

OR and SH performed the study concept and design. OR and OY analyzed the results. TM, MT IJ, KY, FK, and $\mathrm{KH}$ contributed to collecting the cases and performed surgeries. FK and $\mathrm{KH}$ made some meaningful suggestions. All authors have read and approved the final manuscript.

\section{Acknowledgments}

The authors would like to thank MARUZEN-YUSHODO Co., Ltd. (https://kw.maruzen.co.jp/kouseihonyaku/) for the English language editing.

\section{References}

1. Chen DS, Mellman I. Oncology meets immunology: the cancer-immunity cycle. Immunity. 2013;39:110. 
2. Rossille D, Gressier M, Damotte D, Maucort-Boulch D, Pangault C, Semana G, et al. High level of soluble programmed cell death ligand 1 in blood impacts overall survival in aggressive diffuse large B-Cell lymphoma: results from a French multicenter clinical trial. Leukemia. 2014;28:2367-75.

3. Ito M, Oshima Y, Yajima S, Suzuki T, Nanami T, Shiratori F, et al. Is high serum programmed death ligand 1 level a risk factor for poor survival in patients with gastric cancer? Ann Gastroenterol Surg. 2018;2:313-8.

4. Kruger S, Legenstein ML, Rösgen V, Haas M, Modest DP, Westphalen CB, et al. Serum levels of soluble programmed death protein 1 (sPD-1) and soluble programmed death ligand 1 (SPD-L1) in advanced pancreatic cancer. Oncoimmunology. 2017;6:e1310358.

5. Finkelmeier F, Canli Ö, Tal A, Pleli T, Trojan J, Schmidt M, et al. High levels of the soluble programmed death-ligand (SPD-L1) identify hepatocellular carcinoma patients with a poor prognosis. Eur $\mathrm{J}$ Cancer. 2016;59:152-9.

6. Nagato T, Ohkuri T, Ohara K, Hirata Y, Kishibe K, Komabayashi Y, et al. Programmed death-ligand 1 and its soluble form are highly expressed in nasal natural killer/T-cell lymphoma: a potential rationale for immunotherapy. Cancer Immunol Immunother. 2017;66:877-90.

7. Wang L, Wang H, Chen H, Wang WD, Chen XQ, Geng QR, et al. Serum levels of soluble programmed death ligand 1 predict treatment response and progression free survival in multiple myeloma. Oncotarget. 2015;6:41228-36.

8. Zhang J, Gao J, Li Y, Nie J, Dai L, Hu W, et al. Circulating PD-L1 in NSCLC patients and the correlation between the level of PD-L1 expression and the clinical characteristics. Thorac Cancer. 2015;6:534-8.

9. Rossille D, Gressier M, Damotte D, Maucort-Boulch D, Pangault C, Semana G, et al. High level of soluble programmed cell death ligand 1 in blood impacts overall survival in aggressive diffuse large B-Cell lymphoma: results from a French multicenter clinical trial. Leukemia. 2014;28:2367-75.

10. Frigola X, Inman BA, Lohse CM, Krco CJ, Cheville JC, Thompson RH, et al. Identification of a soluble form of $\mathrm{B} 7-\mathrm{H} 1$ that retains immunosuppressive activity and is associated with aggressive renal cell carcinoma. Clin Cancer Res. 2011;17:1915-23.

11. Calderaro J, Rousseau B, Amaddeo G, Mercey M, Charpy C, Costentin C, et al. Programmed death ligand 1 expression in hepatocellular carcinoma: Relationship With clinical and pathological features. Hepatology. 2016;64:2038-46.

12. Ma K, Wei X, Dong D, Wu Y, Geng Q, Li E. PD-L1 and PD-1 expression correlate with prognosis in extrahepatic cholangiocarcinoma. Oncol Lett. 2017;14:250-6.

13. Kruger S, Legenstein ML, Rösgen V, Haas M, Modest DP, Westphalen CB, et al. Serum levels of soluble programmed death protein 1 (sPD-1) and soluble programmed death ligand 1 (SPD-L1) in advanced pancreatic cancer. Oncoimmunology. 2017;6:e1310358.

14. Chang $B$, Huang $T$, Wei $H$, Shen $L$, Zhu D, He W, et al. The correlation and prognostic value of serum levels of soluble programmed death protein 1 (sPD-1) and soluble programmed death-ligand 1 (sPDL1) in patients with hepatocellular carcinoma. Cancer Immunol Immunother. 2019;68:353-63. 
15. Brierley JD, Gospodarowicz MK, Wittekind CH. UICC International Union Against Cancer TNM classification of malignant tumors, 8th edition. New York.

16. Diana A, Wang LM, D'Costa Z, Allen P, Azad A, Silva MA, et al. Prognostic value, localization and correlation of PD-1/PD-L1, CD8 and FOXP3 with the desmoplastic stroma in pancreatic ductal adenocarcinoma. Oncotarget. 2016;7:40992-1004.

17. Royal RE, Levy C, Turner K, Mathur A, Hughes M, Kammula US, et al. Phase 2 trial of single agent Ipilimumab (anti-CTLA-4) for locally advanced or metastatic pancreatic adenocarcinoma. J Immunother. 2010;33:828-33.

18. Chen Y, Li M, Liu J, Pan T, Zhou T, Liu Z, et al. sPD-L1 expression is associated with immunosuppression and infectious complications in patients with acute pancreatitis. Scand $\mathrm{J}$ Immunol. 2017;86:100-6.

19. Zhou J, Mahoney KM, Giobbie-Hurder A, Zhao F, Lee S, Liao X, et al. Soluble PD-L1 as a biomarker in malignant melanoma treated with checkpoint blockade. Cancer Immunol Res. 2017;5:480-92.

20. Akamine T, Takada K, Toyokawa G, Kinoshita F, Matsubara T, Kozuma Y, et al. Association of preoperative serum CRP with PD-L1 expression in 508 patients with non-small cell lung cancer: a comprehensive analysis of systemic inflammatory markers. Surg Oncol. 2018;27:88-94.

\section{Tables}

Table 1 Comparisons of soluble programmed death ligand 1 according to clinicopathological factors and various laboratory data with hepato-biliary-pancreatic cancer

\begin{tabular}{|c|c|c|c|c|}
\hline Variables & & $\begin{array}{l}\text { Number of } \\
\text { patients } \\
(n=119)\end{array}$ & $\begin{array}{l}\text { PDLl level } \\
\text { mean } \pm \text { SD (mg/dl) }\end{array}$ & $P$ value ${ }^{\mathrm{a}}$ \\
\hline \multirow[t]{2}{*}{$\overline{\mathrm{WBC}(/ \mu \mathrm{l})}$} & $<7000$ & 101 & $69.3 \pm 30.4$ & 0.452 \\
\hline & $\geq 7000$ & 18 & $77.5 \pm 34.7$ & \\
\hline \multirow[t]{2}{*}{ Neutrophils (\%) } & $<70$ & 103 & $70.5 \pm 31.6$ & 0.723 \\
\hline & $\geq 70$ & 16 & $70.2 \pm 28.3$ & \\
\hline \multirow[t]{2}{*}{ Lymphocytes (\%) } & $<35$ & 80 & $71.7 \pm 33.6$ & 0.995 \\
\hline & $\geq 35$ & 39 & $68.1 \pm 25.3$ & \\
\hline \multirow[t]{2}{*}{ CRP $(\mathrm{mg} / \mathrm{dL})$} & $<0.3$ & 82 & $71.6 \pm 32.6$ & 0.464 \\
\hline & $\geq 0.3$ & 37 & $68.2 \pm 27.8$ & \\
\hline \multirow[t]{2}{*}{ Albumin $(g / d L)$} & $<3.5$ & 28 & $82.3 \pm 34.9$ & 0.035 \\
\hline & $\geq 3.5$ & 91 & $66.9 \pm 29.1$ & \\
\hline \multirow[t]{2}{*}{ Bilirubin (mg/dL) } & $<1.5$ & 108 & $70.4 \pm 32.1$ & 0.601 \\
\hline & $\geq 1.5$ & 11 & $71.4 \pm 19.7$ & \\
\hline \multirow[t]{2}{*}{ Amylase (U/L) } & $<150$ & 103 & $68.0 \pm 27.4$ & 0.227 \\
\hline & $\geq 150$ & 16 & $86.3 \pm 46.7$ & \\
\hline
\end{tabular}


Table 2 Comparisons of soluble programmed death ligand 1 according to clinicopathological factors and various biomarkers with hepato-biliary-

pancreatic cancer

(A) Hepatocellular carcinoma

\begin{tabular}{lllll}
\hline Variables & & $\begin{array}{l}\text { Number of patients } \\
(n=66)\end{array}$ & $\begin{array}{l}\text { PDLl lerel: } \\
\text { mean } \pm \text { SD }(\mathrm{mg} / \mathrm{dl})\end{array}$ & $P$ value \\
\hline Gender & Male & 46 & $65.4 \pm 26.6$ & 0.201 \\
& Female & 20 & $83.7 \pm 47.9$ & \\
Age (year) & $<65$ & 15 & $79.2 \pm 36.4$ & 0.351 \\
& $\geq 65$ & 51 & $68.5 \pm 34.7$ & \\
Stage & I, II & 33 & $74.8 \pm 39.4$ & 0.533 \\
& III, IV & 33 & $67.1 \pm 30.4$ & \\
AFP (ng/ml) & $\leq 10$ & 37 & $70.5 \pm 33.7$ & 0.969 \\
& $>10$ & 29 & $71.5 \pm 37.5$ & \\
PIVKA-II (ng/ml) & $\leq 40$ & 33 & $72.7 \pm 41.9$ & 0.695 \\
& $>40$ & 33 & $69.1 \pm 27.3$ & \\
\hline
\end{tabular}

\begin{tabular}{|c|c|c|c|c|}
\hline \multicolumn{2}{|l|}{ Variables } & \multirow{2}{*}{$\begin{array}{l}\begin{array}{l}\text { Number of patients } \\
(n=23)\end{array} \\
16\end{array}$} & \multirow{2}{*}{$\begin{array}{l}\text { PDLl level: } \\
\text { mean } \pm \text { SD (mg/dl) }\end{array}$} & \multirow{2}{*}{$\begin{array}{l}P \text { value }{ }^{2} \\
0.688\end{array}$} \\
\hline Gender & Male & & & \\
\hline & Female & 7 & $65.6 \pm 10.5$ & \\
\hline \multirow[t]{2}{*}{ Age (year) } & $<65$ & 5 & $67.4 \pm 26.9$ & 0.371 \\
\hline & $\geq 65$ & 18 & $72.2 \pm 28.6$ & \\
\hline \multirow[t]{2}{*}{ Stage } & I, II & 19 & $73.1 \pm 30.1$ & 0.49 \\
\hline & $\mathrm{mII,IV}$ & 4 & $61.7 \pm 6.2$ & \\
\hline \multirow[t]{2}{*}{$\operatorname{CEA}(\mathrm{ng} / \mathrm{ml})$} & $\leq 5$ & 19 & $70.9 \pm 30.1$ & 0.35 \\
\hline & $>5$ & 4 & $72.3 \pm 14.0$ & \\
\hline \multirow[t]{2}{*}{ CA19-9 (ng/ml) } & $\leq 37$ & 13 & $63.3 \pm 25.5$ & 0.062 \\
\hline & $>37$ & 10 & $81.3 \pm 28.4$ & \\
\hline
\end{tabular}

(C) Pancreatic carcinoma

\begin{tabular}{lllll}
\hline Variables & & $\begin{array}{l}\text { Number of patients } \\
(n=30)\end{array}$ & $\begin{array}{l}\text { PDLl level: } \\
\text { mean } \pm \text { SD }(\mathrm{mg} / \mathrm{dl})\end{array}$ & $P$ value \\
\hline Gender & Male & 14 & $68.8 \pm 20.0$ & 0.901 \\
& Female & 16 & $69.3 \pm 28.0$ & \\
Age (year) & $<65$ & 12 & $63.3 \pm 18.6$ & 0.271 \\
& $\geq 65$ & 18 & $73.0 \pm 27.1$ & \\
Stage & I, II & 19 & $64.7 \pm 24.6$ & 0.149 \\
& III, IV & 11 & $76.7 \pm 22.5$ & \\
CEA (ng/ml) & $\leq 5$ & 17 & $63.6 \pm 15.9$ & 0.346 \\
& $>5$ & 13 & $76.2 \pm 31.2$ & \\
CA19-9 (ng/ml) & $\leq 37$ & 9 & $55.4 \pm 12.4$ & 0.028 \\
& $>37$ & 21 & $74.9 \pm 25.8$ & \\
\hline
\end{tabular}

SD Standard

Whitney U test. Mann- 
Table 3 Univariate and multivariate analysis of risk factors for overall survival with pancreatic cancer

\begin{tabular}{|c|c|c|c|c|c|}
\hline \multirow{2}{*}{ Variables } & & \multirow{2}{*}{$\frac{\text { Univariate }}{P \text { value }}{ }^{\mathrm{a}}$} & \multicolumn{3}{|c|}{ Multivariate } \\
\hline & & & H.R. ${ }^{b}$ & $95 \% \mathrm{CI}^{\mathrm{c}}$ & $\underset{\mathrm{d}}{P}$ value \\
\hline \multirow[t]{2}{*}{ Gender } & Male & 0.429 & & & \\
\hline & Female & & & & \\
\hline \multirow[t]{2}{*}{ Age (year) } & $<65$ & 0.643 & & & \\
\hline & $\geq 65$ & & & & \\
\hline \multirow{2}{*}{$\begin{array}{l}\text { Tumor } \\
\text { loration }\end{array}$} & Head & 0.41 & & & \\
\hline & $\begin{array}{l}\text { Body- } \\
\text { tail }\end{array}$ & & & & \\
\hline \multirow[t]{2}{*}{$\begin{array}{l}\text { Tumor size } \\
(\mathrm{mm})\end{array}$} & $<40$ & 0.342 & & & \\
\hline & $\geq 40$ & & & & \\
\hline \multirow[t]{2}{*}{ UICC $T$ class } & 1,2 & 0.811 & & & \\
\hline & 3,4 & & & & \\
\hline \multirow[t]{2}{*}{ UICC $\mathrm{N}$ class } & 0 & 0.217 & & & \\
\hline & 1 & & & & \\
\hline \multirow[t]{2}{*}{ UICC M class } & 0 & $<0.001$ & 1 & & 0.017 \\
\hline & 1 & & 3.997 & $\begin{array}{l}1.779- \\
17.33\end{array}$ & \\
\hline \multirow[t]{2}{*}{ sPDLl (pg/ml) } & $<81.6$ & 0.001 & 1 & & 0.047 \\
\hline & $\geq 81.6$ & & 3.588 & $\begin{array}{l}1.419- \\
1110\end{array}$ & \\
\hline \multirow[t]{2}{*}{ CEA (ng/ml) } & $\leq \mathbf{5}$ & 0.799 & & & \\
\hline & $>5$ & & & & \\
\hline \multirow{3}{*}{$\begin{array}{l}\text { CAl9-9 } \\
\text { (ng/ml) }\end{array}$} & $\leq \mathbf{3 7}$ & 0.137 & & & \\
\hline & $>37$ & & & & \\
\hline & & & & $\begin{array}{l}\text { a Log. } \\
\text { b. Adju } \\
\text { c Adju } \\
\text { interva } \\
\text { d. Log }\end{array}$ & $\begin{array}{l}\text { test } \\
\text { hazards ratio } \\
95 \% \text { confidence } \\
\text { regression analysis }\end{array}$ \\
\hline
\end{tabular}

Figures 
(A)
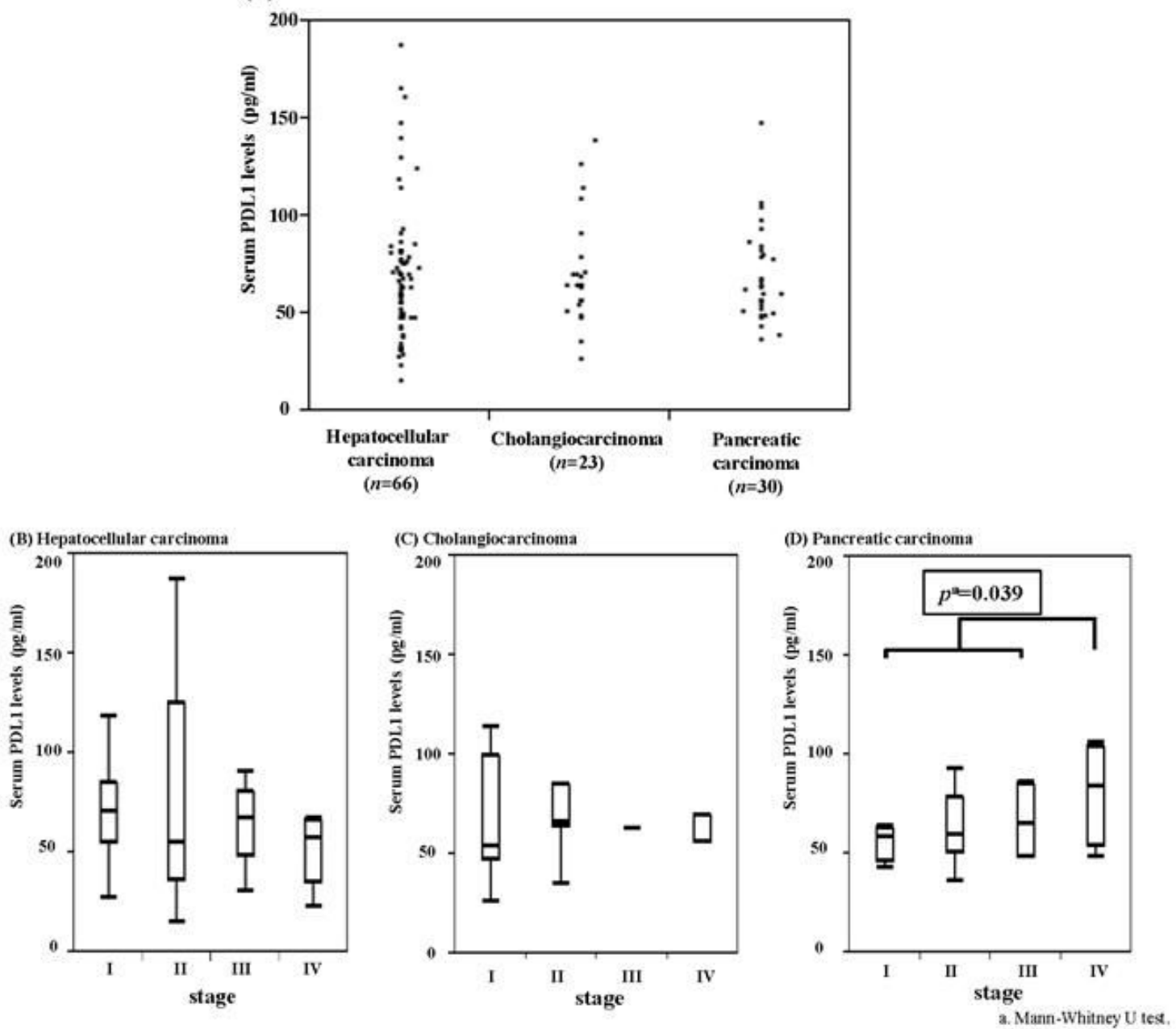

Figure 1

The scatter plot of SPD-L1 level for 66 patients with hepatocellular carcinoma, 23 patients with cholangiocarcinoma, and 30 patients with pancreatic cancer(a). Comparison of sPD-L1 levels according to UICC stages in hepatocellular carcinoma(b), cholangiocarcinoma(c) and pancreatic cancer(d). 


\section{Fig. 2}

(A)

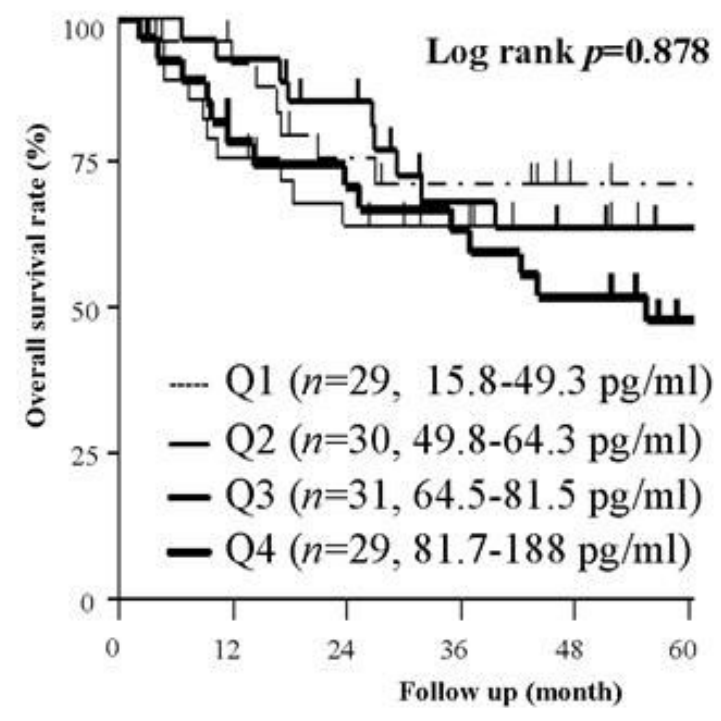

(B)

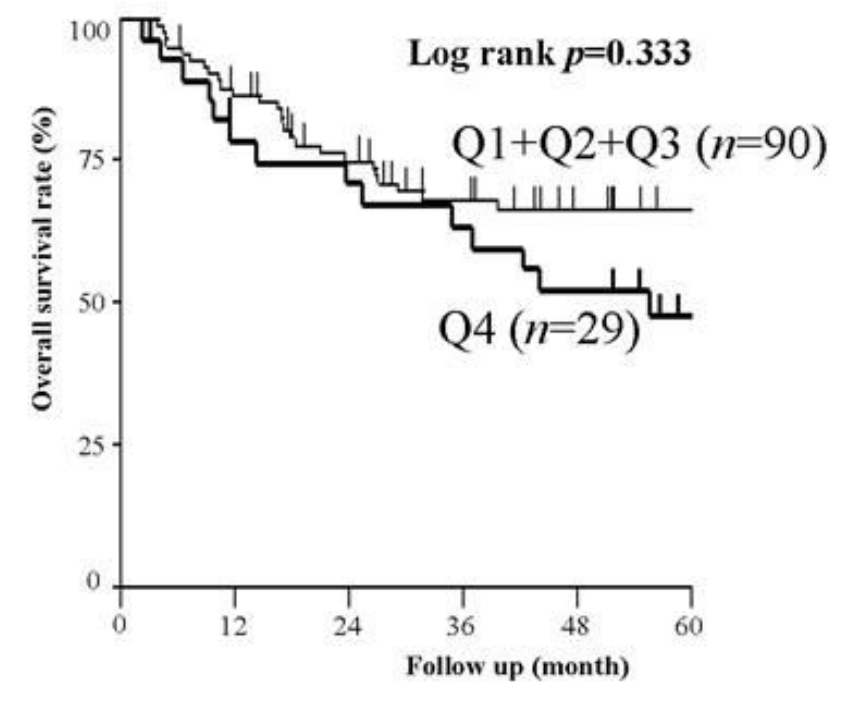

Figure 2

(a) Comparison of overall survivals of the patients with hepato-biliary-pancreatic cancer according to soluble PD-L1 levels classified into four groups (Q1Q2Q3Q4). (b) Comparison of overall survivals according to soluble PD-L1 levels classified into two groups (Q1 + Q2+Q3 vs Q4). Statistical analyses were performed by the Log-Rank test. 


\section{Fig. 3}

(A) Hepatocellular carcinoma

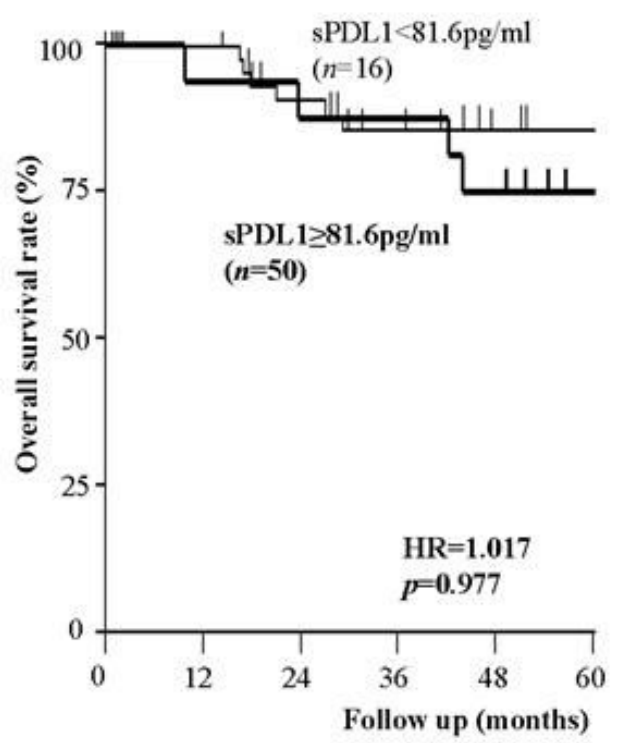

(B) Cholangiocarcinoma

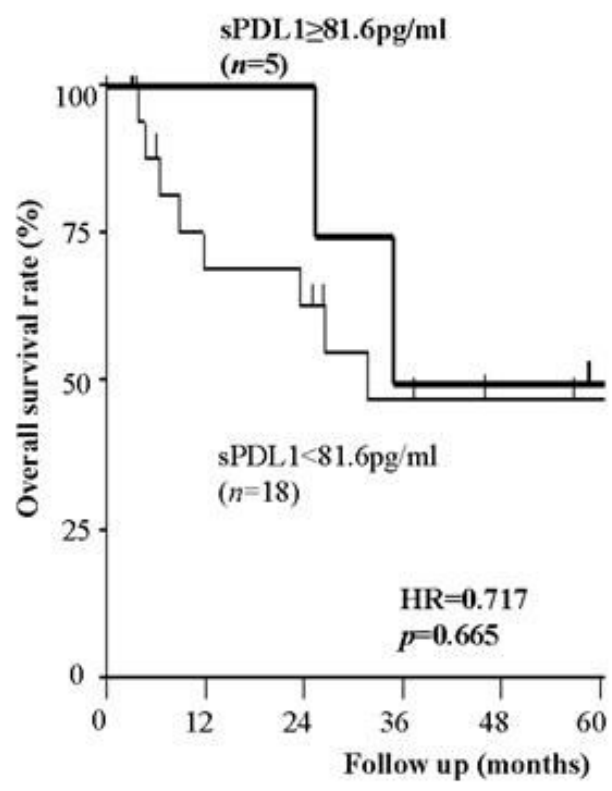

(C) Pancreatic carcinoma

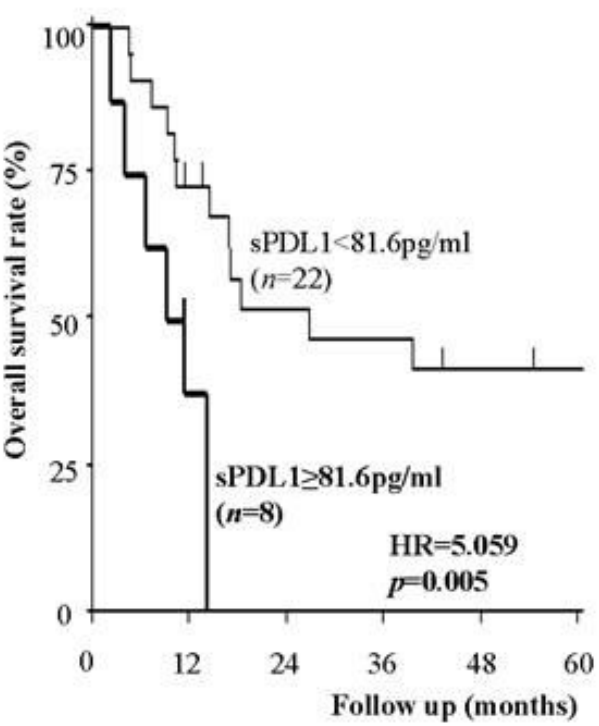

\section{Figure 3}

Comparison of overall survivals according to soluble PD-L1 levels classified into two groups (cut off value: $81.6 \mathrm{pg} / \mathrm{ml}$ ) in hepatocellular carcinoma(a), cholangiocarcinoma(b) and pancreatic cancer(c). Statistical analyses were performed by the Log-Rank test. 